\title{
Sociabilité et socialisation paroissiales : une comparaison franco-allemande
}

\author{
Olivier Bobineau
}

\section{(2) OpenEdition \\ 1 Journals}

Édition électronique

URL : http://journals.openedition.org/assr/3365

DOI : $10.4000 /$ assr.3365

ISSN : $1777-5825$

Éditeur

Éditions de l'EHESS

Édition imprimée

Date de publication : 1 mars 2006

Pagination : 93-114

ISBN : 2-7132-2046-7

ISSN : 0335-5985

Référence électronique

Olivier Bobineau, "Sociabilité et socialisation paroissiales : une comparaison franco-allemande », Archives de sciences sociales des religions [En ligne], 133 | janvier - mars 2006, mis en ligne le 03 mai 2009, consulté le 19 avril 2019. URL : http://journals.openedition.org/assr/3365 ; DOI : 10.4000/ assr.3365 


\section{Olivier Bobineau}

\section{Sociabilité et socialisation paroissiales : une comparaison franco-allemande}

La question de départ est simple : alors qu'Yves Lambert constate au début des années 1980 la fin de la civilisation paroissiale en Bretagne (Lambert, 1985), alors que Danièle Hervieu-Léger examine depuis les années 1990 tant une religion en mouvement qu'une religion en miettes, où chacun en quête d'authenticité se fabrique sur mesure des appartenances et des croyances religieuses (HervieuLéger, 1999, 2001), dès lors, qu'est devenu le paroissien et sa communauté, la paroisse?

Dans le cadre de cet article, il n'est pas question de proposer une vision générale et exhaustive de l'actualité de la paroisse, ni de faire le diagnostic d'une institution locale religieuse. Il s'agit plus simplement de considérer la sociabilité et la socialisation paroissiales à partir de monographies réalisées dans deux paroisses catholiques, l'une en France, l'autre en Allemagne. Parce qu' « on n'explique qu'en comparant", et qu' "une investigation scientifique ne peut donc arriver à sa fin que si elle porte sur des faits comparables et elle a d'autant plus de chances de réussir qu'elle est plus assurée d'avoir réuni tous ceux qui peuvent être utilement comparés » (Durkheim, 1997, p. 1-2), une démarche comparative est retenue étant donné que les deux paroisses présentent des caractéristiques démographiques, socio-économiques et politiques semblables ${ }^{1}$. En outre, le choix de l'Allemagne est opéré car, à notre connaissance, il n'existe pas en langue française de monographie de paroisse allemande catholique contemporaine. Par ailleurs, l'approche est principalement qualitative ${ }^{2}$ dans la mesure où sont appréciées

1. Même si la paroisse française est érigée sur un territoire de 11100 habitants et celle allemande sur un territoire de 5500 habitants, elles ont de nombreux traits communs : BOBINEAU, 2005, p. 37-43 et 161-166.

2. Pour les deux terrains, 31 entretiens exploratoires et 121 entretiens semi-directifs ont été réalisés en plus d'observations participantes ainsi que des "filatures » durant quinze jours auprès de chacun des curés. En outre, une enquête quantitative de pratique dominicale est réalisée à huit jours d'intervalle dans l'une et l'autre paroisse en 2002. Elle permet de disposer d'une photographie à un instant $t$ de la pratique religieuse : à Saint-Yves-des-Monts, ils étaient 389 pratiquants interrogés et 297 à Saint-Martin (voir modalités et résultats de cette enquête : BOBINEAU, 2005). 
de manière approfondie deux configurations paroissiales. En effet, les activités religieuses, "les séquences et les formes d'action » sont prises sous l'angle de la vie au quotidien des acteurs paroissiaux rencontrés en vue de saisir « le religieux en train de se faire en direct » et de découvrir chaque jour " une chaîne d'associations » en train de s'élaborer (Piette, 1999, p. 17 et 57). Aussi, l'analyse proposée ici s'appuie-t-elle essentiellement sur un matériau dont la collecte débute dans les années quatre-vingt-dix et s'achève en 2003. Le premier terrain, la paroisse SaintYves-des-Monts, se trouve dans l'Ouest de la France, dans le diocèse de Laval, en Mayenne. Son curé actuel, Pierre, officie à plein temps depuis 1983. Cette " paroisse nouvelle » regroupe huit communes dans le cadre de la reconfiguration du dispositif paroissial entamée depuis le début des années quatre-vingt-dix dans les diocèses français (Palard, 1999). La seconde paroisse, Saint-Martin (paroisse érigée sur une seule commune), est en Souabe bavaroise (région de l'Allgäu), dans le diocèse d'Augsbourg ; elle est placée sous la responsabilité de Paul ${ }^{3}$ depuis 1994.

Après avoir mis en évidence différents types de sociabilité paroissiale communs aux deux paroisses, cet article précisera en quoi la socialisation paroissiale se différencie à Saint-Yves-des-Monts et Saint-Martin. Cela permettra d'identifier deux processus spécifiques de socialisation à l'œuvre : celui de personnalisation dans la paroisse mayennaise et celui de communautarisation dans son homologue bavaroise.

\section{Une sociabilité paroissiale plurielle et commune aux deux paroisses}

Qu'entendre tout d'abord par sociabilité paroissiale ? On peut partir de la définition de la sociabilité du sociologue Roger Levasseur : "L'espace de relations intermédiaires qui se situe au-delà des nécessités élémentaires de l'existence (se nourrir, se vêtir, se loger, travailler), de la vie privée et des rapports avec les intimes (famille), et en deçà des pouvoirs institués (Église, État, entreprise) » (Levasseur, 1990, p. 9). Même si la paroisse fait partie d'un pouvoir institué (l’Église), elle n'en demeure pas moins un espace intermédiaire dans lequel se développent des relations qui manifestent « l'aptitude générale d'une population à vivre intensément les relations publiques» (Agulhon, 1977, p. 7). La paroisse peut être donc conçue comme un cercle, un réseau reliant, rassemblant des acteurs qui choisissent de s'inscrire de manière plus ou moins formelle dans un cadre social déterminé. Ainsi, la sociabilité paroissiale peut se définir comme le développement de relations sociales d'inter-connaissance et d'inter-reconnaissance engendrées au sein du dispositif paroissial.

3. Comme il l'a été demandé et afin de préserver leur anonymat, les prénoms, noms des personnes interrogées ont été modifiés ainsi que les noms des deux paroisses, des mouvements directement rattachés à celles-ci. 
À Saint-Yves-des-Monts et à Saint-Martin, la sociabilité paroissiale revêt plusieurs formes. Quatre parmi elles peuvent être distinguées à titre principal et correspondent à quatre profils de fidèles (fidèle entendu comme personne attestant socialement d'un lien avec la paroisse). Une telle approche de la sociabilité paroissiale relève d'une démarche idéaltypique au sens de Weber, c'est-à-dire d'une construction conceptuelle accentuant par la pensée certains éléments de la réalité (Weber, 1992, p. 172-173).

\section{Sociabilité paroissiale occasionnelle et fiddèe saisonnier}

La première forme de sociabilité est occasionnelle, c'est-à-dire que des personnes participent, à l'occasion, à une activité, une manifestation ou un service de la paroisse. Cela peut être lors d'une communion, d'une confirmation, d'un mariage d'un membre de la famille (ou son propre mariage) ou encore d'une sépulture d'un proche, d'un voisin. Durant un instant, celui de la cérémonie religieuse, la personne se déplace et rencontre les paroissiens ; il s'agit là d'un contact ponctuel lors d'événements qui rythment le calendrier cultuel de la communauté. C'est aussi la participation momentanée à des manifestations proposées par la paroisse. Lors de la fête paroissiale, d'un regroupement de l'une des associations rattachées à la paroisse ou d'une conférence, d'une rencontre, d'un débat organisés par des paroissiens, des personnes viennent assister seules ou en famille. Si cette sociabilité est minimale dans la mesure où elle est fragmentée et irrégulière, elle n'en demeure pas moins importante pour la paroisse car elle sociabilise, le temps d'une manifestation, des personnes ayant peu de contacts avec l'Église et ouvre la communauté paroissiale à d'autres réalités culturelles au travers de rencontres imprévues.

La sociabilité paroissiale occasionnelle s'appuie sur le fidèle saisonnier. Il s'agit, d'une part, de personnes qui, pour un sacrement ou un rite de passage, s'adressent à la communauté paroissiale et sollicitent un service spirituel. Ces "paroissiens de passage » se font chrétiens l'instant d'un "son de cloche » comme le fait observer le prêtre français. Ces consommateurs de sacrements, croyants ou non, demandent et usent d'un rite procuré par le curé et l'assemblée des paroissiens ; ils le font par tradition ou pour faire " plaisir à un membre de leur famille» le plus souvent, note le curé bavarois. Comme l'écrit Gabriel Le Bras, au sujet des « conformistes saisonniers ", ces pratiquants sont :

Ceux qui se portent ou que l'on porte (et c'est alors leur famille qui les engage ou simplement témoigne) à l'église du village pour recevoir le baptême, la première communion, le sacrement de mariage et les honneurs de la sépulture (Le Bras, 1976, p. 167).

Le fidèle saisonnier est, d'autre part, celui qui vient à une manifestation ou une activité proposée par la paroisse. Il se déplace alors pour une raison précise : il apprécie une messe qu'il juge dynamique, recherche une ambiance, il veut 
ponctuellement prendre part à l'activité d'une association satellite ${ }^{4}$ de la paroisse. Des personnes sont ainsi amenées à fréquenter la communauté paroissiale le temps d'un événement : dans la paroisse allemande, on les appelle des "visiteurs ", dans la paroisse mayennaise, des "paroissiens de cœur».

\section{Sociabilité paroissiale ordinaire et fidèle pratiquant}

Par ordinaire, il faut comprendre la sociabilité développée par des acteurs au travers d'activités paroissiales liturgiques et sacramentelles régulières. Ces activités, séculaires et habituelles pour une paroisse, sont autant d'occasions pour des fidèles de tisser régulièrement des liens au travers d'événements cultuels. Plus que les messes de semaine peu fréquentées, le point d'orgue de la communauté sont les messes du week-end, où les paroissiens peuvent se réunir et discuter ensemble.

À Saint-Yves-des-Monts, les membres des équipes liturgiques ${ }^{5}$ en profitent pour échanger les nouvelles locales, se retrouver entre voisins, entre amis mais aussi avec des personnes que l'on apprend à connaître. À Saint-Martin, la préparation de la messe est une occasion d'échanges et de rencontres entre les enfants de chœur, les lecteurs, le sacristain et le prêtre. Au sortir de la messe, les fidèles se réunissent dans l'église ou sur le parvis pour échanger quelques minutes. Dans les deux paroisses, une petite troupe se forme : "les discussions vont bon train ", « on parle de tout et de n'importe quoi » nous signale-t-on. On donne des nouvelles de la famille, on discute politique et religion selon l'actualité locale, nationale et internationale; le prêtre va de groupe en groupe de discussions. On en profite pour se donner des rendez-vous et s'inviter. C'est un temps de rassemblement de fidèles et d'interconnaissance. En plus des messes du week-end, il y a également les grands rendez-vous liturgiques de l'année: Noël, Pâques, la Pentecôte, la Toussaint et aussi la fête de la paroisse. Si l'observance n'est plus celle du temps de la civilisation paroissiale, la régularité de l'offre liturgique permet à de multiples acteurs d'échanger, de partager et de confronter ponctuellement des regards et points de vue. Autant d'échanges parfois furtifs, dont la fréquence rythme le lien social local et produit une catalyse sociale à laquelle sont invités les fidèles et les passants.

Cette sociabilité paroissiale s'appuie sur le fidèle pratiquant. Ce dernier conçoit la paroisse comme un lieu de culte où il vient pratiquer en famille et/ou individuellement sa foi. Régulièrement, au moins une fois par semaine, il se déplace

4. À la discrétion des acteurs religieux locaux, il existe des associations, des groupements par affinités qui gravitent autour de la paroisse : ces satellites regroupent des croyants et noncroyants.

5. Dans cette paroisse, 32 équipes liturgiques de laïcs rassemblent chacune 3 laïcs provenant des huit communes de la paroisse. À tour de rôle, chaque équipe choisit les chants et les lectures selon un cycle liturgique prédéfini. Par ailleurs, l'équipe liturgique se charge de la décoration de l'église, de trouver un organiste et un animateur. 
pour vivre en communauté un temps de prière et de partage. Le fidèle pratiquant va à la messe parce qu'il s'y sent bien et qu'il considère que cette pratique est nécessaire et vitale pour l'épanouissement de sa personne. Par choix et non par obligation, par envie et non "pour faire plaisir ", il vient assister à la célébration dominicale.

À l'heure du "zapping religieux ", la volonté de pratiquer "contre vents et marées ${ }^{6}$ dans une communauté précise est le véritable ressort du fidèle pratiquant. Il tient à "sa " messe, il fait en sorte de ne pas la manquer quoi qu'en pensent les autres. S'il est de plus en plus rare d'aller régulièrement à la messe, si cela semble "étrange aujourd'hui d'y aller » ou " marginal ", voire " dévalorisant " comme le déclarent certains paroissiens, le fidèle résiste et pratique de la sorte sa croyance. En reprenant l'approche de l'identité ${ }^{7}$ développée par Manuels Castells, le fidèle pratiquant est porteur d'une certaine «identité résistance » au sens où il fait partie "des acteurs qui se trouvent dans des positions ou des conditions dévalorisées et/ou stigmatisées par la logique dominante » et qu'il résiste en affirmant des " principes étrangers ou contraires à ceux qui imprègnent les institutions de la société » (Castells, 1999, p. 18). S'il ne s'investit pas nécessairement dans les autres activités de la paroisse (services paroissiaux, satellites), il participe au demeurant aux cérémonies religieuses selon des modalités personnelles. En effet, le fidèle pratiquant aujourd'hui à Saint-Yves-des-Monts et SaintMartin s'accommode de la célébration : il a non seulement " la tête ailleurs en pleine célébration " mais également " chante, participe quand il veut, selon son humeur » remarquent les curés. Il arrive parfois en retard ou part avant le chant d'envoi parce qu'il reçoit des amis, prépare le repas ou doit s'affairer à d'autres activités. Il dispose d'une palette d'expressions liturgiques (chants, gestes, déplacements) qu'il mobilise selon ses besoins et ses aspirations. De plus, le fidèle pratiquant est effacé et relativement discret : il est souvent en retrait, dans l'ombre des collaborateurs salariés et de bénévoles plus investis dans les structures de la paroisse. Son adhésion s'exprime donc principalement au travers d'une pratique régulière tout en rendant très occasionnellement des services, des "coups de main ».

\section{Sociabilité paroissiale associative et fidèe militant}

Le qualificatif "associatif » est à entendre au sens large, caractérisant les groupements volontaires qui se réunissent régulièrement pour poursuivre un objectif déterminé. Cette sociabilité se déploie sous de multiples formes et nourrit le tissu social local en fédérant les acteurs locaux, croyants et non croyants.

6. Il s'agit d'un chant pratiqué à Saint-Yves-des-Monts.

7. Manuel Castells définit comme suit l'identité : "processus de construction de sens à partir d'un attribut culturel, ou d'un ensemble cohérent d'attributs culturels, qui reçoit priorité sur toutes les autres sources ", un même individu, ou un même acteur collectif pouvant en avoir plusieurs (Castells, 1999, p. 17). 
Autour de l'épicentre paroissial, trois cercles associatifs peuvent être distingués selon la finalité du regroupement et les relations entretenues avec la structure paroissiale.

- Le premier cercle rassemble des équipes émanant directement de la paroisse et assurant en quelque sorte un service extraordinaire aux fidèles et à la population locale. Ces activités ne sont pas directement liées aux activités ordinaires, à l'agenda liturgique; elles résultent d'orientations et de choix pastoraux. À Saint-Yves-des-Monts, les " équipes de proximité " ${ }^{8}$, les équipes d'éveil à la foi, de la catéchèse familiale, de la catéchèse des 8-11 ans génèrent des liens entre fidèles, familles et habitants. À Saint-Martin, les «cercles des mères ", les groupes de missions, les chorales, les équipes organisant le travail en direction des enfants et des adolescents, le port de la communion et les visites aux malades produisent également des liens entre les fidèles, les familles et les habitants.

- Le deuxième cercle est composé de mouvements (principalement d'action catholique spécialisée) représentés dans la paroisse. Ils sont les correspondants locaux de mouvements nationaux, parfois internationaux. Si ces mouvements ont leur logique propre et font appel à des aumôniers extérieurs à la paroisse, celle-ci en constitue un échelon fédérateur stable et se trouve alors prise dans une chaîne institutionnelle supra-paroissiale. Concrètement, elle est le lieu d'accueil des membres de ces mouvements et met à leur disposition une logistique, à laquelle prennent part des paroissiens. De façon régulière, des fidèles se regroupent au sein de ces mouvements sur le territoire de la paroisse et proposent des activités ouvertes à tous. Ils interviennent sur des champs spécifiques selon des objectifs déterminés indépendamment des choix pastoraux paroissiaux. De manière générale, les membres et publics de ces groupements sont des personnes âgées. À SaintYves-des-Monts, s'il y a le Mouvement Eucharistique des Jeunes (une équipe de cinq jeunes en 2002), les autres mouvements (Chrétiens en Monde Rural : six couples; Mouvement des Chrétiens Retraités : 14 femmes; Hospitalières de Lourdes: 10 membres; le Secours Catholique: 22 bénévoles; la Fraternité Chrétienne des Malades : 65 membres) rassemblent essentiellement des personnes du troisième âge et de sexe féminin. À Saint-Martin, des mouvements équivalents sont aussi le fait de personnes retraitées: Behinderten-Seelsorge (mouvement s'occupant de personnes handicapées: 13 membres en 2002); Sozialdienst Katholischer Franen (service catholique social pour les femmes : 25 membres); $K A B$ (action catholique ouvrière : 40 membres); Kolping (catholicisme social : 70 membres).

- Le dernier cercle repose sur l'activité des satellites de la paroisse. Ils sont en lien affinitaire avec la paroisse et développent leurs actions selon des objectifs,

8. Par l'expression "équipes de proximité ", les acteurs désignent les activités suivantes réalisées par des bénévoles : les équipes liturgiques, l'accueil des personnes demandant le baptême et le mariage à l'église, l'accueil des familles en deuil, le fleurissement et l'entretien de l'église, la distribution du bulletin paroissial. 
des moyens co-définis par des acteurs locaux. Regroupant aussi bien des habitués de la communauté paroissiale que des habitants distants de l'Église, ces satellites constituent une interface au niveau local entre l'institution religieuse et la société. À cet égard, ils sont le vivier associatif de la paroisse, où se croisent de manière plus ou moins régulière les habitants. Laissés à la discrétion des laïss, les satellites sont d'une certaine manière le fleuron de la paroisse, "la fierté paroissiale " comme nous le font comprendre bon nombre de personnes. Perçus comme dynamiques, ils sont à mi-chemin entre l'institution ecclésiale, qui conserve toujours un œil sur eux, et la société civile. Ils rassemblent en priorité un public jeune : jeunes animateurs, jeunes couples, enfants et adolescents. À Saint-Martin, le Bistro ${ }^{9}$, mais aussi le travail œcuménique sont le fruit d'une population jeune, tout comme Bâtir Tous Demain ${ }^{10}$ et Action Humanitaire ${ }^{11}$ à Saint-Yves-des-Monts.

Ces trois cercles concourent à tisser autour de la paroisse un réseau, une toile d'interconnaissance sociale. Ces groupes volontaires reposant sur l'implication forte de subjectivités individuelles mettent en commun des sensibilités, des appartenances, des conceptions de l'action collective. Au regard des profils variés composant ces groupes affinitaires, la sociabilité associative de la paroisse développe une politique de «la main tendue », une ouverture aux réalités locales mais aussi internationales.

La sociabilité paroissiale associative prend appui sur le fidèle militant, lequel prend part à un ou plusieurs cercles ci-dessus précisés. Contrairement au fidèle pratiquant, le fidèle militant n'a pas nécessairement une pratique régulière. La pratique est même très occasionnelle pour les jeunes fidèles militants. Ce fidèle conçoit avant tout la paroisse comme un centre d'activités religieuses et un organe du développement local nécessaire à la vie communale. Pour lui, la paroisse est une communauté porteuse de projets témoignant de valeurs humaines et spirituelles au service de la collectivité. Acteur critique, il considère que, comme

9. Placé sous la responsabilité des enfants de chœur depuis 1968, ce café (jus de fruits, bières, pizzas, gâteaux bavarois) se situe sous la sacristie de l'église. Lieu de rencontre pour les jeunes, et décoré par eux, il est un espace d'accueil ouvert à tous les paroissiens pouvant recevoir jusqu'à 100 personnes lors d'une soirée ou d'une animation proposée par des membres de SaintMartin. En plus des 80 enfants de chœur qui fréquentent régulièrement ce café, des paroissiens de tout âge viennent parfois s'y restaurer.

10. Créée en 1984, cette association a pour objet de « rejoindre et accompagner les jeunes dans la découverte de la foi, en les aidant à faire leur choix et à prendre leur place au service des autres dans l'Église et dans la société d'aujourd'hui ". Elle organise plusieurs camps d'été pour des jeunes selon trois tranches d'âge : 9-11 ans, 12-14 ans et 15-17 ans. En août 2003, près de 250 jeunes, 40 animateurs (dont Pierre, d'autres prêtres, des séminaristes, des enseignants et des étudiants) et 15 intendants partent en France et à l'étranger.

11. Association fondée en 1981, Action Humanitaire avec ses 25 membres cherche d'une part à faire connaître les Pays En Développement en proposant des conférences qui présentent d'autres horizons culturels aux habitants de la paroisse. D'autre part, elle collecte des fonds pour financer des projets de développement défendus par une personne qui est en lien avec la communauté paroissiale. 
l'écrit Jean-Marie Donegani à propos du pluralisme religieux et politique dans le catholicisme contemporain : "être catholique, c'est s'engager pour construire un monde »(Donegani, 1993, p. 242).

Le fidèle militant est un animateur/fédérateur de projets. Convaincu de la nécessité d'agir sur le monde au nom de sa foi, il participe, élabore des activités et parfois innove en termes d'animation. S'il doute et remet en question souvent sa croyance, il attend beaucoup de la confrontation et de la rencontre avec d'autres : il recherche des "signes", des occasions de confirmer/valider sa foi. Il est en outre un animateur/fédérateur de réseaux. Ancré dans le paysage local, ayant d'autres responsabilités associatives, il connaît les habitants et est en lien avec des acteurs locaux : élus, commerçants, artisans, responsables associatifs et syndicaux... Enfin, le fidèle militant est un passeur d'idées. En lien avec l'institution paroissiale mais également reconnu par une partie de la population locale, il dispose d'une légitimité pour faire entendre ses opinions et conceptions, qu'elles concernent l'Église ou la société. Il assure des médiations entre la communauté paroissiale et les habitants non croyants. En s'inspirant du vocabulaire de Manuel Castells, disons que le fidèle militant est le porteur de «l'identité projet » de la communauté paroissiale, au sens où il fait partie des acteurs qui cherchent à construire une identité dont la visée est de transformer une partie de la structure sociale (Castells, 1999, p. 18 et 88 ).

\section{Sociabilité paroissiale institutionnelle et fidde «consacré» 12}

Cette sociabilité recouvre les liens et échanges plus ou moins formels qui ont lieu lors des rencontres et réunions des organes institutionnels paroissiaux reconnus par le droit canon. Cette armature institutionnelle se constitue d'un conseil pastoral $^{13}$ (discussion des orientations), d'un conseil des affaires économiques (gestion et administration des biens de la paroisse) et d'une équipe pastorale (mise en œuvre des orientations). Dans chacune des paroisses étudiées, près de 25 paroissiens se rassemblent tout au long de l'année au sein de ces différents organes institutionnels. Cette sociabilité, plus formalisée en apparence que les trois précédentes, se développe au fil de discussions et d'échanges. Ces réunions sont l'occasion parfois de partager des repas, souvent de prendre un verre. On retrouve des paroissiens qui développent leurs points de vue sur l'Église; ils parlent également de l'actualité, locale, nationale et internationale tout en donnant

12. Il est fait référence à la figure sociologique du croyant «consacré " mise en avant par Jean-Paul Willaime dans un article portant sur le christianisme : «Le christianisme : une religion de l'avenir de la religion?" (Willaime, 1999, p. 227-246). Il ne s'agit pas ici des "laïcs consacrés » qui ; sans être religieux, ont prononcé des vœux par devant leur évêque.

13. Par "pastoral " ou "pastorale ", l’Église entend exprimer, depuis Vatican II, «les rapports de l'Église et du monde, de l'Église et des hommes d'aujourd'hui » (note explicative de la Constitution pastorale de l'Église dans le monde de ce temps). 
des nouvelles de leur famille respective. Des liens d'amitié, du fait d'affinités et d'engagements communs, se tissent de la sorte.

Toutefois, si ces personnes se connaissent bien et s'apprécient, le lien de travail qui les réunit a ses exigences, ses contraintes et les amènent à débattre, se confronter. D'un côté, la mise en commun des préoccupations, des idées, des sensibilités est finalisée : accomplir une mission commune, à savoir décider, orienter et mettre en œuvre les activités de la paroisse. De l'autre, des désaccords, des enjeux de pouvoir et de reconnaissance sociale émergent : qui fait quoi ?, pourquoi cette personne et pas une autre ?, avec quels moyens ?, quel message faire passer ? Prenons le cas concret de l'organisation de la fête patronymique qui relève, dans chacune des deux paroisses, de la compétence des membres du conseil pastoral, du conseil des affaires économiques et de l'équipe pastorale. Il faut en choisir tout d'abord le thème central. Au fil des réunions, les uns souhaitent que cette journée festive, véritable « vitrine de la communauté » comme ils le disent, soit consacrée à une question d'actualité internationale, à un document publié par le pape ; les autres ambitionnent de soulever une question de société (avec des intervenants extérieurs) ou souhaitent s'interroger sur la place de la communauté paroissiale dans la société ; les derniers désirent simplement "passer un bon moment ensemble ». Si l'alchimie met du temps à se réaliser, les arbitrages finissent par dégager une thématique commune. Puis, une fois le sujet choisi, il faut mobiliser les autres paroissiens, préparer et coordonner les activités de la fête, en organiser les détails matériels et faire fonctionner la logistique et les réseaux en vue d'informer la population. Quand la fête est passée, il faut apprécier ce qui vient de se produire et de se vivre afin de faire mieux la fois prochaine. Autant d'occasions et de réunions qui mêlent disputes et accords, tensions et jeux d'influence au sein de la communauté paroissiale.

Personnes cooptées, diacres, bénévoles, devenus pour quelques-uns d'entre eux des amis du prêtre, ils soutiennent concrètement l'activité religieuse à Saint-Yvesdes-Monts. À Saint-Martin, ce sont des élus, des bénévoles, des collaborateurs salariés par la paroisse qui constituent les architectes de l'activité paroissiale. Pour les deux curés rencontrés, cette coopération des laïcs doit aller de pair avec " une bonne entente sincère », une "vraie solidarité » entre les membres de ces organes. Si les échanges, les liens ne sont pas bons ou entretenus, la "dynamique » et «la source d'énergie » de la paroisse sont mises à mal. De fait, la sociabilité institutionnelle est un moteur de la communauté paroissiale; elle est synonyme de vitalité et de bon fonctionnement s'appuyant sur la responsabilisation effective des laïcs. Insuffisante, mais nécessaire, la sociabilité institutionnelle assure à Saint-Martin et Saint-Yves-des-Monts le développement des activités religieuses.

Ce type de sociabilité est mis en œuvre par le fidèle pratiquant qui peut se définir comme le militant dévoué à la "cause » et comme reconnu légitime par la communauté paroissiale pour agir en son nom. 
En premier lieu, il est un militant convaincu non seulement de la cause paroissiale mais plus généralement de la force du message du christianisme. Se déclarant "dévoué à la paroisse ", il mobilise ses compétences, ses talents au service du fonctionnement et de la mise en œuvre d'activités religieuses en suivant des formations continues (théologique, pastorale, animation liturgique...). Il passe du temps et se donne pour que tout se passe bien. Il est un pratiquant régulier et présent aux manifestations de la paroisse. On peut compter sur lui quand il le faut, c'est-à-dire souvent. En outre, au nom de sa foi, il s'engage au sein de diverses associations, pas nécessairement confessionnelles. Dans la mesure du possible, il témoigne au fil de ses responsabilités associatives et dans son travail de sa conception de «la solidarité chrétienne », du "service chrétien »; il se veut d'une certaine manière "dévoué à l'humanité au quotidien, fidèle en cela au Christ » pour reprendre des expressions entendues. À la suite de JeanPaul Willaime, on peut dire de ce fidèle qu'il est « consacré » dans la mesure où il fait partie:

de ces femmes et ces hommes qui, comme religieuses et religieux, prêtres et pasteurs, laïcs professionnels et bénévoles engagés dans tel mouvement d'aide sociale ou dans telle institution caritative d'inspiration chrétienne, de ces femmes et ces hommes qui, par leur engagement quotidien et en vivant quelquefois eux-mêmes au milieu des plus démunis, témoignent de cette dimension essentielle du christianisme qu'est le "souci du plus petit de mes frères " (Willaime, 1999, p. 239).

Précisément, ce fidèle s'implique en prenant des responsabilités au service du "plus petit ", des autres et de sa paroisse. Acteur au cœur de son Église, acteur au sein de la société, il cherche à donner une image renouvelée de la paroisse qu'il connaît bien et qu'il aime.

En second lieu, le fidèle «consacré » est reconnu légitime pour représenter la communauté paroissiale et mettre en œuvre des actions en son nom. Qu'il soit élu, coopté, choisi pour être membre d'un organe de l'armature institutionnelle, il est apprécié parce qu'il apporte quelque chose à la paroisse. Il est respecté par le reste des fidèles qui reconnaissent son activité et son courage ainsi que son degré d'investissement et d'implication dans les services et ouvrages de la communauté. Il peut par conséquent représenter la paroisse lors de manifestations publiques locales ou de rencontres infra-diocésaines. Cependant, cette reconnaissance sociale ne l'empêche pas d'être critique à l'égard de l'institution romaine. Bien au contraire, plus il s'inscrit dans la communauté paroissiale, plus il prend des responsabilités, plus il devient critique à l'égard de "Rome". De ce point de vue, il est le défenseur et l'avocat de «sa " paroisse face à tout représentant d'une autre structure ecclésiale. En reprenant les termes de Manuel Castells, il est le porteur de « l'identité légitimante » de la communauté paroissiale au sens où il soutient l'influence, « la domination de l'institution » qu'il représente sur les autres (Castells, 1999, p. 18). Il cherche également à réconcilier le croyant et la structure paroissiale en inscrivant un témoignage de foi dans un dispositif officiel local; il est en quelque sorte l'acteur singulier du rapprochement entre 
la tradition et la vie actuelle, " la religion des pères " et " la religion des frères " (Hervieu-Léger, 1997, p. 318).

Finalement, aux côtés du fidèle saisonnier, moins impliqué, les fidèles pratiquant, militant et "consacré ", participent selon les modalités de leur choix à la construction identitaire de leur communauté et de la société locale, articulant les identités "résistance ", " projet » et « légitimante" (Castells, 1999). Mais, cette catégorisation n'est pas figée au regard des parcours de vie des paroissiens. Les personnes rencontrées passent d'un profil à l'autre selon les périodes, le temps disponible, leurs "envies". Ainsi, on est un temps durant (une année par exemple) fidèle militant, puis "consacré ", pour devenir fidèle occasionnel et inversement. Comme en témoigne le curé de Saint-Yves-des-Monts : "Les paroissiens s'investissent vraiment comme ils l'entendent. Cela peut être "tout" d'un seul coup : les associations, l'équipe pastorale, puis un arrêt pour des raisons familiales, professionnelles; parfois aussi, il y en a qui commencent doucement puis s'affirment progressivement. Y en a qui partent et qui reviennent, qui font leur temps et laissent la place à d'autres! ». Au gré de cette mobilité de l'investissement des paroissiens ${ }^{14}$, la sociabilité paroissiale se tisse et demeure en quelque sorte une réponse localisée et multiforme, au sein de l'institution catholique, à «l'insociable sociabilité des hommes » pour reprendre le philosophe de Königsberg (Kant, 1985, p. 192).

\section{Des processus de socialisation paroissiale opposés : personnalisation, communautarisation}

Selon Tocqueville, "pour qu'il y ait société, et, à plus forte raison que cette société prospère, il faut que tous les esprits des citoyens soient toujours rassemblés et tenus ensemble par quelques idées principales» (Tocqueville, 1992, p. 519). Dès lors, "les hommes ont donc un intérêt à se faire des idées bien arrêtées sur Dieu, leur âme, leurs devoirs généraux envers leur Créateur et leurs semblables ; car le doute sur ces premiers points livrerait leurs actions au hasard et les condamnerait en quelque sorte au désordre et à l'impuissance» (idem, p. 531). C'est dans cette optique que les paroissiens s'interrogent sur le point suivant: comment transmettre des « idées principales » sur « Dieu », les « devoirs généraux » envers le "Créateur » et leurs « semblables » aux jeunes générations ? Voici donc soulevée la question de la socialisation que l'on peut définir, à la suite d'Henri Mendras, comme «le mécanisme par lequel la société transmet ses normes, ses

14. Sur l'investissement des paroissiens dans l'action collective au sein de la paroisse, on peut retrouver un terrain d'application du «cycle privé/public » mis en évidence par Albert O. Hirschman. En effet, le sociologue américain observe " un mouvement d'aller et retour entre la vie publique et la vie privée » chez nos contemporains selon leurs intérêts, attentes et désirs, déceptions et satisfactions procurées par les sphères privée et publique (HIRSCHMAN, 1995, p. 22). 
valeurs et ses croyances à ses membres» (Mendras, 1996, p. 242). Quelle en est alors la forme prise à Saint-Yves-des-Monts et à Saint-Martin ? De cette comparaison, il ressort que la place qu'occupe la communauté paroissiale diverge sensiblement selon le terrain considéré. Si l'on définit le processus de socialisation paroissiale comme le dispositif social par lequel la communauté paroissiale transmet ses normes, ses valeurs et ses croyances, on peut avancer l'hypothèse que ce processus est opposé dans les deux paroisses.

\section{Saint-Yves-des-Monts : de la foi personnelle vers l'institution}

À Saint-Yves-des-Monts, les paroissiens témoignent d'une foi, qui est aussi bien un véritable sentiment, une raison de vivre, qu'un questionnement. Force et fragilité, convictions et doutes, tout cela se croise et coexiste dans le même temps chez la même personne. Les récits personnels entendus lors des entretiens illustrent l'importance du rôle joué par la foi comme clé de relecture du parcours de vie : chacun dévoile ses interrogations, ses tourments mais aussi ses projets. La foi est alors un révélateur du vécu, une manière de sentir et de voir intimement fondée, dans une société où "il ne fait pas bon d'être catholique » comme le déclare une jeune paroissienne. La prière individuelle est en outre une pratique importante, protéiforme, plus ou moins régulière. Les personnes interrogées reconnaissent la réaliser dans leur vie quotidienne selon les «envies »; chacun s'exprime largement sur la question dès lors qu'une relation de confiance se tisse.

Pour les Mayennais rencontrés, la paroisse est une famille, un lieu chaleureux de proximité qui mobilise les énergies individuelles. L'instance assurant la synergie des paroissiens est l'établissement de la paroisse nouvelle, à la fois projet et défi selon la question récurrente : "Comment vivre ensemble la présence de Dieu au travers de nouveaux moyens et de nouvelles activités ?». Paroisse nouvelle dont les paroissiens veulent donner une autre, et tant qu'à faire, une bonne image dans une société où prolifère le "croire " et où il s'agit d'être bien perçu face aux autres concurrents. La difficulté réside donc dans le fait de témoigner de la foi en un Dieu, en référence à une tradition, qu'il s'agit de "traduire " auprès de personnes, qui n'ont plus la «culture catho». Les plus jeunes des paroissiens tentent en particulier de présenter à leurs connaissances et amis l'image d'une institution paroissiale ouverte et vivante dans un contexte d' "exculturation » du catholicisme français, c'est-à-dire une situation marquée non seulement par le rétrécissement avéré de l'influence de l'Église dans la société, mais aussi par la « déliaison » progressive entre la culture commune et le tissage catholique issu de l'histoire (Hervieu-Léger, 2003, p. 97).

Certes, les paroissiens de Saint-Yves-des-Monts témoignent de leur foi en s'engageant dans le développement local. De ce point de vue, la paroisse peut être, selon l'expression employée par une bénévole, «le pain du village » dans 
la mesure où elle est un vivier de citoyens, un espace créateur de solidarités développant du lien social et proposant des regards alternatifs. Toutefois, ce n'est pas tant la paroisse en tant qu'institution qui est reconnue, mais bien des acteurs catholiques qui s'investissent dans la vie politique et sociale. Contrairement à ce qui se passe dans la paroisse allemande, il n'y a pas à Saint-Yves-des-Monts de liens officialisés entre la paroisse et la municipalité, il n'y a que des relations et des affinités interpersonnelles entre les différents acteurs locaux.

Dans ce contexte, la socialisation paroissiale opérée à Saint-Yves-des-Monts prend appui sur la motivation, la foi personnelle pour transmettre et diffuser les valeurs, les codes, les croyances auxquels tiennent les différents fidèles. Une telle transmission est donc centrée sur la découverte et le développement d'un socle spirituel qui peut progressivement s'incarner dans une adhésion à l'institution paroissiale. De sorte qu'en Mayenne, le fidèle assume et découvre d'abord sa foi (Bobineau, 2005) et, dans un second temps, s'associe à la communauté pour s'investir éventuellement au sein de l'institution paroissiale, dont il deviendra un jour, peut-être, le porte-parole.

\section{Saint-Martin : de l'institution vers la foi personnelle}

Deux observations peuvent aider à mieux saisir la place et le rôle joué par la tradition catholique dans le processus de socialisation s'opérant dans la région où se trouve la paroisse Saint-Martin, l'Allgäu bavarois.

D'une part, la visite d'une exposition publique à Memmingen, ville proche de la paroisse, met en évidence le poids de la tradition catholique dans la région. Le thème de cette manifestation est le jardin. On s'attend à voir les innovations en matière de culture botanique ou les derniers outils mis au point pour jardiner ou encore à découvrir les initiatives de quelques jardiniers talentueux. Bref, nous sommes prêts à mieux saisir la fibre bavaroise environnementale. Mais, ce n'est pas ce qui frappe l'attention tout au long de l'exposition. Sur 39 stands, 18 se réfèrent directement à la tradition catholique en mettant en lumière des acteurs (prêtres, religieux, personnes en train de prier), des symboles monumentaux (crucifix, maquettes d'église, presbytère, cimetières) ou des représentations relevant de son patrimoine historique (paradis, poissons, statues de saints et de la vierge...). Même le dernier stand de cette exposition, qui est celui, officiel, du Ministère de l'environnement, de la protection des eaux et forêts présente un grand panneau qui attire l'attention du visiteur au centre de l'emplacement. En ce sens, des photos de personnalités politiques et religieuses sont appuyées par un unique commentaire : «Nous soutenons et reconnaissons nos origines historiques et religieuses ». Une telle manifestation ouverte au public, financée par des collectivités locales, légitime en la mettant en scène, la transmission d'un référent culturel catholique. 
La seconde observation a trait à l'école, lieu par excellence de la diffusion des codes, des valeurs d'une société. Pour la communauté paroissiale de SaintMartin, les cours de religion catholique qui ont lieu à l'école publique ${ }^{15}$ constituent un moyen privilégié de socialisation religieuse. En effet, l'enseignant de ce cours peut être le curé de la paroisse, son assistant paroissial ou tout autre membre de la communauté qui a reçu une formation de "professeur de religion». En conséquence, les cours de religion sont un temps particulier pour transmettre les valeurs et croyances auxquelles sont attachés les paroissiens. À cet égard, un panneau retient l'attention dans la salle de classe destinée au cours de religion catholique de l'école publique. Il est accroché à côté du tableau et, à plusieurs reprises, il est montré du doigt par les professeurs de religion. Voici son contenu :

Règles de conversation:

1) Nous écoutons celui qui parle!

2) Nous le laissons finir de parler!

3) Nous levons la main!

4) Nous attendons jusqu'à ce que tous écoutent!

5) Nous restons sur le sujet!

6) Nous respectons d'autres opinions!

7) Nous ne faisons jamais d'attaques personnelles !

8) Nous participons!

Comme le font remarquer les trois enseignants avec lesquels nous discutons de ce règlement, ces règles sont essentielles à plusieurs titres. D'une part, elles affirment le "nous" : il s'agit de "règles pour bien vivre dans la communauté que constitue la classe » souligne un enseignant. D'autre part, elles mettent en avant la volonté de « considérer l'autre comme son égal en l'écoutant, en évitant des querelles inutiles" rappelle un autre. De ce point de vue, les professeurs tiennent à afficher et faire respecter ce règlement en cours de religion catholique parce qu' "il ne constitue pas autre chose que les valeurs auxquelles nous croyons en tant que catholiques : respect de l'autre et vie en communauté » conclut l'un d'entre eux.

Ces deux observations mettent en valeur un point essentiel : la prégnance et la reconnaissance de la religion catholique en tant qu'instance de socialisation

15. La Loi fondamentale de la République allemande donne des prérogatives aux Églises en matière d'enseignement. Selon l'article 7-3, «l'instruction religieuse est une matière d'enseignement régulière dans les écoles publiques ", elle est " dispensée conformément aux principes des communautés religieuses, sans préjudice du droit de contrôle de l'État ». Concrètement, l'enseignement religieux est une matière obligatoire et notée au même titre que les autres tout au long de la scolarité de l'élève jusqu'au baccalauréat. La prise en charge de cet enseignement dans les écoles publiques est assurée par les ministères de l'Éducation, de la Science et de l'Art de chaque «Land» en lien avec les autorités religieuses. L'assistance y est obligatoire pour tous, mais à partir de 14 ans, l'élève peut choisir entre un cours de religion et un cours d'éthique. Les cours durent de deux à trois heures par semaine selon les niveaux et font l'objet d'un examen obligatoire au baccalauréat (KOENIG, 2005, p. 131-141). 
intergénérationnelle en Allgäu. Phénomène, qui plus est, reconnu et entretenu par les membres de Saint-Martin.

De fait, la socialisation paroissiale repose, en premier lieu, sur une forte implantation locale et institutionnalisée de la religion catholique, incarnée par de multiples structures, équipements et personnes salariées par la paroisse. En effet, dans le cadre du modèle partenarial allemand entre les Églises et l' État (Partnerschaft), scellé par la Loi fondamentale, les Églises peuvent disposer de moyens financiers, fonciers et de ressources humaines en grand nombre à tous les niveaux : fédéral, régional, local (Willaime, 2003). Concrètement, la paroisse Saint-Martin est propriétaire en propre des biens et bâtiments qu'elle utilise (l'église, le presbytère, les salles de réunions, le Bistro et la salle paroissiale) alors que la paroisse française est simplement affectataire des édifices religieux. En outre, elle administre deux établissements scolaires qui lui sont rattachés (un jardin d'enfants, une école spécialisée pour enfants en difficultés). Pour faire fonctionner tout ceci, le curé de Saint-Martin, à la différence de son homologue français, est entouré de collaborateurs salariés, rémunérés principalement par le diocèse d'Augsbourg et la commune. Le prêtre est ainsi à la tête d' " une véritable entreprise » de 22 personnes salariées ${ }^{16}$, ce qui représente 15 emplois à plein temps. Ce dispositif encadre et intègre le fidèle dès son plus jeune âge. Dès lors, il ne s'agit pas tant d'une intégration dans la communauté paroissiale se manifestant par une pratique spirituelle volontaire ou l'affirmation d'une foi personnelle que d'une «intégration communautaire par le dispositif paroissial », comme le signifie le prêtre bavarois. Cette intégration se déroule au fil d'un cycle de vie personnel encadré par la communauté catholique : l'enfant est scolarisé dans une école où il reçoit une instruction religieuse; à l'adolescence, il participe à des mouvements et associations catholiques très actifs en Bavière; lors de ses études ou de sa formation professionnelle, il peut s'inscrire dans un établissement catholique (université, école professionnelle); devenu adulte, il peut être salarié du diocèse (celui d'Augsbourg emploie 13000 personnes), dans le même temps, ses enfants sont scolarisés dans une école gérée par une paroisse et ses parents pris en charge par des foyers et maisons de retraite catholiques. Comme le note ce professeur dans un lycée professionnel au sujet des jeunes adultes : «Ils quittent la paroisse pour des raisons d'études ou professionnelles; ils quittent leur famille d'origine et... reviennent quand ils en ont une nouvelle!». Une paroissienne commente également : "Les enfants reviennent à l'Église après 30 ans quand ils ont des enfants à leur tour et qu'ils les scolarisent dans un jardin d'enfants catholique ; ils sont critiques mais ils donnent les valeurs qu'ils ont reçues. Pour cela, les institutions catholiques présentes un peu partout sont une chance pour les

16. Trois groupes de salariés peuvent être distingués à Saint-Martin : « l'équipe rapprochée du curé » (les deux assistants paroissiaux, le sacristain, l'organiste, les deux secrétaires, le séminariste) ; "l'équipe scolaire » des deux établissements rattachés à la paroisse (deux directrices, quatre éducatrices, cinq puéricultrices); «l'équipe d'entretien» (trois personnes). 
jeunes ». En second lieu, la socialisation paroissiale se manifeste dans la mise en œuvre d'activités et de services communautaires, dont l'une des finalités est de transmettre et d'entretenir les croyances religieuses. Précisément, le travail en direction des enfants, des adolescents ou les cercles des mères, sans oublier le port de la communion et les visites aux malades, constituent une offre intergénérationnelle animée par des paroissiens et proposée aux habitants de la paroisse.

Par conséquent, en reprenant les analyses du sociologue allemand Karl Gabriel, on peut dire que la paroisse Saint-Martin développe tant "une logique interne d'intégration sociale de ses membres » qu' « une logique externe d'influence du système social » tout en veillant à la "transmission de ses sources et origines » : elle est à la fois une structure localement intégrée et intégrante (Gabriel, 1999, p. 31-32).

Cependant, une telle socialisation, évidente et permanente pour les acteurs locaux, n'en fait pas moins l'objet de critiques. Certains paroissiens notent le fait que des fidèles considèrent la communauté avant tout comme refuge institutionnel en délaissant le développement d'une pratique spirituelle personnelle. Des fidèles précisent : "Saint-Martin est pour des paroissiens une institution qu'il faut utiliser et respecter avant d'être un espace de vie spirituelle ». Le curé ajoute, non sans un certain sens de l'ironie: "Nous avons affaire à quelques "fonctionnaires du lieu" ${ }^{17}$ qui sont bien loin de mettre en avant leur foi dans la communauté paroissiale mais l'utilité, la fonction que cela procure! ». De telles remarques renvoient à une réalité que nous pouvons saisir ainsi : dans le cadre du modèle partenarial État/Églises, la communauté paroissiale est une institution légitime accompagnant le fidèle depuis sa petite enfance, durant sa scolarité et sa vie familiale d'adulte jusqu'à la maison de retraite de sorte qu'il peut découvrir et assumer éventuellement sa foi au fil de sa fréquentation des institutions paroissiales et catholiques.

En schématisant, on dirait que le fidèle de la paroisse, à Saint-Martin, passe de l'institution à une pratique éventuelle de sa foi personnelle alors que le fidèle, à Saint-Yves-des-Monts, passe de sa pratique de foi personnelle à une inscription éventuelle dans l'institution.

\section{Saint-Yves-des-Monts : le processus de personnalisation}

Les paroissiens manifestent à plusieurs reprises lors de prières, de sépultures ou d'activités paroissiales un processus de socialisation spécifique. Ainsi, la prière $\mathrm{du}$ paroissien en assemblée dominicale est vécue et exprimée en lien avec une

17. Avec cette formule, Paul fait volontairement un clin d'œil à la traduction française du livre d'Eugen Drewermann Fonctionnaires de Dieu, publié aux éditions Albin Michel en 1993 (le titre en allemand est Kleriker, Psychogramm eines Ideals, publié en 1989). 
communauté d'appartenance : la prière collective soutient la prière individuelle. Entre le « je » et le «nous » communautaire, une relation se tisse et relève de l'articulation de l'individu à sa communauté. Tel est le cas lors des baptêmes, mariages, confirmations, durant lesquels les prières mettent en valeur des personnes et sont, dans le même temps, portées par la communauté paroissiale et adressées à Dieu. Pour les sépultures, un dispositif (fidèles de la paroisse et famille du défunt) se met en place qui, durant la cérémonie, entretiendra une tension entre, d'un côté, la valorisation de la personne décédée, y compris sur le plan émotionnel (lectures de poèmes écrits par le défunt, utilisation de musiques contemporaines, de photos, de diapositives), et, de l'autre, la reconnaissance sociale de l'événement douloureux par la communauté, la famille, les amis. La sépulture est ce moment privilégié où chacun, réuni en communauté, se questionne quant à son rapport à la mort, à "l'ailleurs" et à "l'autre » : on reconnaît communautairement et affectivement une expérience perçue comme éminemment subjective et douloureuse : la mort d'un proche (Déchaux, 2001, p. 87). Enfin, la fête annuelle paroissiale, les soirées animées par les associations satellites, les réunions des organes de la paroisse ou le pèlerinage annuel sont autant d'occasions durant lesquelles des acteurs sociaux, des personnes mettent en scène et en sens leurs visions du monde dans le cadre de la communauté paroissiale tout en faisant référence à Dieu.

Comment alors comprendre et caractériser conceptuellement en terme de socialisation ce matériau collecté à Saint-Yves-des-Monts? En s'appuyant sur les travaux de Mauss (Mauss, 1997) et de Mounier pour lequel « la vie personnelle n'est pas repli sur soi, mais mouvement vers et avec autrui, vers et sur le monde matériel, vers un au-dessus et un au-delà de l'acquis " (Mounier, 1962, p. 230), nous définissons le processus de socialisation suivant: le processus de personnalisation est l'ensemble des phénomènes sociaux se reproduisant avec une certaine régularité et articulant trois dimensions : le "je " agissant, repérable dans les manifestations et actions des acteurs sociaux; le "nous " structurant incarné par une communauté ${ }^{18}$; le dépassement et le décentrement en référence à une altérité, religieuse ou non. Dans notre recherche, le "je » agissant se retrouve chez les différents paroissiens, le "nous » est la communauté paroissiale SaintYves-des-Monts, le dépassement et le décentrement en référence à une altérité s'opèrent selon la représentation d'un Dieu. Un tel processus caractéristique de la paroisse mayennaise se différencie toutefois de celui observé dans la paroisse bavaroise.

18. La communauté est ici entendue au sens de «communalisation » de Max Weber : « Nous appelons "communalisation" une relation sociale lorsque, et tant que, la disposition de l'activité sociale se fonde - dans le cas particulier, en moyenne ou dans le type pur - sur le sentiment subjectif (traditionnel ou affectif) des participants d'appartenir à une même communauté " (WEBER, 1995, p. 78). 


\section{Saint-Martin : un processus de communautarisation}

Un samedi soir, avec deux jeunes paroissiens, nous allons au cinéma. Avant la séance, une réclame retient l'attention. Il s'agit de celle d'une limonade américaine adressée aux plus jeunes spectateurs ; son slogan est bref : Teile ! (Partage !). De retour en France, nous découvrons la réclame pour le même produit au cinéma, mais elle se conclut par un slogan très différent : "N'écoute que toi ! »... Une telle comparaison publicitaire serait anecdotique si elle n'illustrait pas entre la France et l'Allemagne la différence de positionnement social et culturel de l'individu en société ou en communauté. Progressivement, l'enquête à Saint-Martin apporte des éléments de réponse à cette combinaison du «je » et du «nous ». Pour reprendre l'approche développée par Louis Dumont au sujet de "l'idéal de la Bildung ", " une dualité qui est à la fois caractéristique et, à première vue curieuse "s'observe chez les Allemands avec "d'un côté, on voit une survivance tranquille, aux Temps modernes, de la communauté, c'est-à-dire d'un sentiment, d'une orientation holiste, qui se traduit dans la vie de tous les jours par le penchant quasi-proverbial à obéir, la soumission spontanée aux autorités politiques et sociales » et «d'un autre côté, il y a un développement intérieur fort prononcé de l'individualité, une intériorité jalousement cultivée » (Dumont, 1991, p. 35).

Cette dualité entre l'approfondissement d'une intériorité spirituelle individuelle et un "dévouement au tout " (Dumont, 1985, p. 43) s'exprime tout au long des activités et services paroissiaux proposés à Saint-Martin. En étudiant les chants des livrets utilisés par la paroisse Saint-Martin et ceux de Saint-Yvesdes-Monts (le livret de Saint-Yves-des-Monts comporte 116 chants, celui de Saint-Martin, 93), on remarque une plus ou moins grande présence des pronoms personnels « je » et « nous » ainsi que des adjectifs possessifs « mon, ma, mes » et «notre, nos ». De fait, les adjectifs possessifs et pronoms personnels de la première personne du pluriel sont cinq fois plus nombreux que ceux de la première personne du singulier dans les chants utilisés par les paroissiens de Saint-Martin; dans les chants de Saint-Yves-des-Monts, les adjectifs possessifs et pronoms personnels de la première personne du pluriel sont quasiment aussi nombreux que ceux de la première personne du singulier ${ }^{19}$. Un tel écart met en évidence le rôle spécifique que joue le «nous » à Saint-Martin au travers de chants et prières pratiqués par la communauté paroissiale. Pour ce qui est de la prière, et à la différence des paroissiens mayennais, ceux de Saint-Martin, pour une large part, reconnaissent ne pas prier individuellement de leur côté ; ils le font principalement lors de rassemblements et assemblées communautaires ${ }^{20}$.

19. Prenons un exemple: les chants du livret utilisé par la communauté de Saint-Martin comprennent cinq fois plus de références au «Nous Te rendons grâce » qu'au « Je Te rends grâce " alors que ceux de la paroisse Saint-Yves-des-Monts comportent autant de références à l'une et à l'autre de ces prières.

20. Sur 60 paroissiens interrogés, 9 font état d'une prière personnelle en dehors de la prière communautaire du dimanche. Parmi eux, se trouvent cinq femmes (dont l'assistante paroissiale) et quatre hommes (dont le sacristain, le séminariste et le prêtre). 
Lors des sépultures, le rite sobre est préfixé et reconduit un ordre social et religieux dans lequel la communauté de Saint-Martin est au premier plan : une valorisation du rôle structurant de la communauté est assurée par le prêtre qui a pour fonction d'attester le bon fonctionnement du rite de passage devant tous.

De manière générale, l'encadrement prégnant $\mathrm{du}$ « nous » se retrouve en filigrane dans d'autres activités paroissiales : les festivités de la semaine sainte, la fête de l'épiphanie les premiers jours du mois de janvier, les camps d'été organisés pour les enfants de chœur, les rencontres œcuméniques... L'ensemble de ces dispositifs et services produisent et mettent en avant la médiation de l'institution communautaire, jugée comme fondamentale pour pratiquer sa foi et vivre en société. Prenons un dernier exemple : un soir de mars 1999, nous assistons à une réunion du comité liturgique de la paroisse. Composé de sept personnes, ce comité se réunit cinq fois par an pour faire le point sur les célébrations. Ce soir-là, l'ordre du jour porte sur la communion des fidèles. La discussion part de l'interrogation de l'un des participants sur l'opportunité de dire deux fois "Amen » lors de l'eucharistie. En effet, les fidèles lors de la communion sont invités à former deux ou trois cercles au pied de l'autel. Quand le petit cercle s'est formé, le donneur de communion ou le prêtre met l'hostie dans les mains croisées de chaque membre du groupe et reprend sa place au centre du cercle. Puis, il prononce une parole de l'Évangile du jour et tous communient au corps du Christ. Cette démarche est répétée jusqu'à ce que l'ensemble des personnes ait communié. Le fidèle peut dire une première fois "Amen " lorsqu'il reçoit dans ses mains le pain consacré et une seconde fois quand, après la parole évangélique, il mange l'hostie avec les autres membres du cercle. Aussi, comme le demande une paroissienne, faut-il dire une ou deux fois "Amen " ? Après des échanges et propos à forte teneur théologique, une solution est finalement trouvée : dire une première fois « Amen » pour recevoir personnellement le corps du Christ, le dire une seconde fois pour manifester avec d'autres et dans le même temps son appartenance à la communauté paroissiale.

Dès lors, au regard du matériau collecté en Souabe bavaroise, nous définissons le processus de communautarisation comme suit: l'ensemble des phénomènes sociaux se reproduisant avec une certaine régularité, dans lesquels le "nous" institutionnalisé ${ }^{21}$ encadre et structure le "je » agissant, repérable dans les manifestations et actions des acteurs sociaux, en référence à une altérité, religieuse ou non. Le "nous " institutionnalisé s'incarne dans la paroisse Saint-Martin, le " je » agissant est celui des paroissiens et la référence à une altérité se réalise au travers de la représentation d'un Dieu.

21. Par institutionnalisé, il faut entendre ce qui « dans une société donnée, prend la forme d'un dispositif organisé, visant au fonctionnement ou à la reproduction de cette société, résultant d'une volonté originelle (acte d'instituer) et d'une adhésion, au moins tacite, à sa légitimité supposée » (BONTE, IZARD, 2000, p. 376). 
Au total, même si les deux paroisses révèlent une sociabilité commune, le processus de socialisation n'en diffère pas moins. D'un côté, à Saint-Martin, un processus de communautarisation encadre et structure le fidèle depuis sa plus jeune enfance jusqu'à sa mort, au sein d'une institution religieuse (Bobineau, 2005), légitime politiquement et reconnue localement. La paroisse est dans ce cas précis une "forme sociale institutionnalisée " (Gabriel, 2000, p. 346), bien intégrée dans la société locale parce qu'intégrant et socialisant de façon continue plusieurs générations. De l'autre, à Saint-Yves-des-Monts, un processus de personnalisation articule le parcours individuel de foi du fidèle à une communauté, dans laquelle il s'investit éventuellement selon ses « envies » et aspirations. La paroisse est ici un centre d'activités spirituelles qui diffuse localement des croyances et des valeurs en s'appuyant sur le bénévolat; il s'agit d'une forme sociale affinitaire cherchant à socialiser sur le plan spirituel de manière durable les nouvelles générations.

Olivier BOBINEAU

Groupe, Sociétés, Religions, Laïcités

\section{Bibliographie}

AgulHon Maurice, Le Cercle dans la France bourgeoise (1810-1848). Étude d'une mutation de sociabilité, Paris, Armand Colin, 1977.

Bobineau Olivier, Dieu change en Paroisse. Une comparaison franco-allemande, Rennes, Presses Universitaires de Rennes, 2005.

Bonte Pierre, IzARD Michel, dirs, Dictionnaire de l'ethnologie et de l'anthropologie, Paris, PUF, [1991], 2000.

Castells Manuel, Le pouvoir de l'identité. L'ère de l'information, volume II, Paris, Fayard, [1997], 1999.

Déchaux Jean-Hugues, "Un nouvel âge du mourir: "la mort en soi” ", Recherches Sociologiques, vol. 32, $\mathrm{n}^{\circ}$ 2, 2001, p. 79-100.

Dumont Louis, «L'idée allemande de liberté selon Ernst Troeltsch », Esprit, n 4-5, 1985, p. $40-50$.

Dumont Louis, Homo Aequalis II. L'idéologie allemande. France-Allemagne et retour, Paris, Gallimard, 1991.

DonÉGANI Jean-Marie, La liberté de choisir, pluralisme religieux et pluralisme politique dans le catholicisme français contemporain, Paris, Presses de la FNSP, 1993.

DurKheIM Émile, Le suicide. Étude de sociologie, Paris, PUF, [1897], 1997.

Gabriel Karl, « Modernisierung als Organisierung von Religion », in Michael KRÜGGeler, Karl GABRIEL, Winfried GEBHARDT, dirs, Institution, Organisation, Bewegung, Sozialformen der Religion im Wandel, Opladen, Leske et Budrich, 1999, p. 19-37.

GABRIEL Karl, "Déchristianisation et sécularisation : aspects sociologiques et statistiques ", in Paul Colonge, Rudolf Lill, dirs, Histoire religieuse de l'Allemagne, Paris, Cerf, 2000, p. 333-348. 
Hervieu-Léger Danièle, "Le croyant et l'institution ", in Jacques Palard, dir., Le gouvernement de l'Église catholique. Synodes et exercice du pouvoir, Paris, Cerf, 1997, p. 313-322.

Hervieu-Léger Danièle, Le Pèlerin et le Converti. La religion en mouvement, Paris, Flammarion (coll. "Champs »), 1999.

Hervieu-Léger Danièle, La religion en miettes ou la question des sectes, Paris, CalmannLévy (coll. "Essai société »), 2001.

Hervieu-LéGer Danièle, Catholicisme, la fin d'un monde, Paris, Bayard (coll. "Questions en débat »), 2003.

Hirschman Albert-O, Bonheur privé, action publique, Paris, Fayard, [1982], 1995.

KANT Emmanuel, Euvres philosophiques, volume II, Paris, Gallimard (Bibliothèque La Pléiade), 1985.

KoEnIG Matthias, "L'État-nation allemand à l'épreuve des mutations de l'enseignement religieux » in Jean-Paul Willaime, dir. (avec la collaboration de Séverine Matthieu), Des maîtres et des dieux. Écoles et religions en Europe, Paris, Belin, 2005, p. 131-141.

LAmbert Yves, Dieu change en Bretagne, Paris, Cerf, 1985.

LE Bras Gabriel, L'église et le village, Paris, Flammarion, 1976.

LeVasseur Roger, dir., De la sociabilité. Spécificités et mutations, Montréal, Les Éditions du Boréal, 1990.

MAuss Marcel, "Une catégorie de l'esprit humain : la notion de personne, celle de "moi” " [1938], Sociologie et anthropologie, Paris, PUF, [1950], 1997, p. 331-362.

Mendras Henri, Éléments de sociologie, Paris, Armand Colin, [1975], 1996.

Mounier Emmanuel, Euvres de Mounier, tome III, 1944-1950, Paris, PUF, 1962.

PietTe Albert, La Religion de près. L'activité religieuse en train de se faire, Paris, Métailié, 1999.

PALARD Jacques, «Les recompositions territoriales de l'Église catholique entre singularité et universalité, territorialisation et centralisation ", Archives de Sciences Sociales des Religions, 107, p. 55-75, 1999.

REY Alain, Le Robert, dictionnaire historique de la langue française, Paris, Dictionnaires Le Robert, [1992], 2000.

Tocqueville Alexis de, Euvres, volume II, Paris, Gallimard (Bibliothèque La Pléiade), 1992.

Weber Max, «L'objectivité de la connaissance dans les sciences et la politique sociales » [1904], Essais sur la théorie de la science, Paris, Presses Pocket (coll. "Agora »), 1992, p. $117-201$.

Weber Max, Économie et société, les catégories de la sociologie, [1922], tome I, Paris, Presses Pocket, 1995.

Willaime Jean-Paul, «Le christianisme : une religion de l'avenir de la religion ? ", in René RÉmOND, dir., Les grandes inventions du christianisme, Paris, Bayard, 1999, p. 227 246.

Willaime Jean-Paul, "La réunification de l'Allemagne et ses incidences religieuses », in Fabienne RandaXHe, Valentine ZubER, dirs, Laïcité-démocraties. Des relations ambiguës, Turnhout, Brepols, 2003, p. 31-45. 
I 4 - ARCHIVES DE SCIENCES SOCIALES DES RELIGIONS

Mots-clés : sociabilité paroissiale, socialisation paroissiale, France-Allemagne, lien social

\section{Résumé}

Alors que l'on observe l'effondrement de la civilisation paroissiale depuis les années soixante, cet article propose, à partir d'une comparaison franco-allemande, un examen approfondi et actuel de la sociabilité et de la socialisation paroissiales. Après avoir mis en évidence et caractérisé les différents types de sociabilité communs aux deux paroisses (l'une se trouve dans le diocèse de Laval, l'autre dans le diocèse d'Augsbourg), il s'agira de voir en quoi et comment la socialisation paroissiale se produit de manière différenciée dans ces deux communautés.

\section{Abstract}

It is common knowledge that parochial civilisation has been in decline since the 1960s. This article proposes a thorough contemporary investigation into parish sociability and socialization based on a Franco-German comparison. After highlighting and defining the different types of sociability shared by both parishes - one located in the diocese of Laval, the other in the diocese of Augsburg - this study will examine the ways in which*1 parochial socialization differs in the two communities.

\section{Resumen}

Mientras que se observa la disolución de la civilización parroquial desde los años sesenta, este artículo propone, a partir de una comparación franco-alemana, un examen profundo y actual de la sociabilidad y de la socialización parroquiales. Luego de haber puesto en evidencia y caracterizado los diferentes tipos de sociabilidad comunes a las dos parroquias (una en la diócesis de Laval, otra en la diócesis de Augsbourg), se tratará de ver en qué y cómo la socialización parroquial se produce de manera diferenciada en estas dos comunidades. 